\title{
Genomic-wide analysis and prediction of genes involved in biosynthesis of polysaccharide and bioactive secondary metabolites in high-temperature- tolerance of wild Flammulina filiformis
}

\author{
Juan Chen ( $\nabla$ kibchenjuan@126.com ) \\ Jia-Mei Li \\ Chinese Academy of Medical Sciences \& Peking Union Medical College Institute of Medicinal Plant Development \\ Yan-Jing Tang \\ Institute of Medicinal Plant Development, Chinese Academy of Medical Science \\ $\mathrm{Ke} \mathrm{Ma}$ \\ Institute of Microbiology Chinese Academy of Sciences \\ Bing Li \\ Chinese Academy of Medical Sciences \& Peking Union Medical College Institute of Medicinal Plant Development \\ Xu Zeng \\ Chinese Academy of Medical Sciences \& Peking Union Medical College Institute of Medicinal Plant Development \\ Xiao-Bin Liu \\ Kunming Institute of Botany Chinese Academy of Sciences \\ Yang Li \\ Chinese Academy of Medical Sciences \& Peking Union Medical College Institute of Medicinal Plant Development \\ Zhu-Liang Yang \\ Kunming Institute of Botany Chinese Academy of Sciences \\ Wei-Nan Xu \\ Fujian Agriculture and Forestry University \\ Bao-Gui Xie \\ Fujian Agriculture and Forestry University \\ Shun-Xing Guo \\ Chinese Academy of Medical Sciences \& Peking Union Medical College Institute of Medicinal Plant Development
}

Chinese Academy of Medical Sciences \& Peking Union Medical College Institute of Medicinal Plant Development https://orcid.org/0000-0002-5407-8239

\section{Research article}

Keywords: Edible mushroom, Gene cluster, Polysaccharide, Secondary metabolites, Gene expression, High-temperature-tolerance

Posted Date: August 23rd, 2019

DOI: https://doi.org/10.21203/rs.2.13424/v1

License: (9) (7) This work is licensed under a Creative Commons Attribution 4.0 International License. Read Full License

Version of Record: A version of this preprint was published at BMC Genomics on October 17th, 2020. See the published version at https://doi.org/10.1186/s12864-020-07108-6. 


\section{Abstract}

Background: Flammulina filiformis (=Asian "F.velutipes") is a popular commercial edible mushroom. Many bioactive compounds such as polysaccharides and sesquiterpenoids with medicinal effects have been isolated and identified, but their biosynthesis and regulation in molecular level is unclear. In this study, we sequenced the genome of the wild strain F. filiformis Liu 355, predicated the biosynthetic gene clusters (BGCs) and profiled these genes expression between wild and cultivar strains and among different development stages of the wild strain of F. filiformis by a comparative transcriptomic analysis. Results: The results revealed that the genome of the F. filiformis was $35.01 \mathrm{M} \mathrm{bp}$ in length and annotated with 10396 gene models. 12 putative terpeniod gene clusters were predicted, 12 sesquiterpenes synthase genes belonged to four different groups and two type I PKS (polyketide synthase) gene clusters were identified from F. filiformis genome. The gene number related terpeniod biosynthesis is higher in wild strain (119 genes) than cultivar strain (81 genes) and most of them are up regulated in primodium and fruiting body of the wild strain, while PKS genes are usually up-regulated in the mycelium of wild strain. Moreover, genes encoding UDP-glucose pyrophosphorylase and UDP-glucose dehydrogenase involved in polysaccharide biosynthesis have relative high transcripts both in mycelium and fruiting bodies of F. filiformis. Conclusions: We identified candidate genes involved in the biosynthesis of polysaccharide and terpenoid bioactive compounds and profiled these genes expression during the development of F. filiformis. This study expends our knowledge for understanding the biology of F. filiformis and provides valuable data for elucidating the secondary metabolism regulation of the special strain of F. filiformis.

\section{Background}

Flammulina filiformis (= Asian F. velutipes),, a species endemic to Asian region, as known as Enokitake, winter mushroom or golden needling mushroom, belongs to the family Physalacriaceae, Agaricales [1]. Recent new phylogenetic result based on multi-gene markers and morphological comparison demonstrated that "F.velutipes" in eastern Asia is not identical to the European winter mushroom F. velutipes, and should be treated as a separate species, namely F. filiformis, including all cultivated strains of "F. velutipes" in East Asia and those from South Korea and Japan with genome sequences [2]. Thus, we used the name "F. filiformis" instead of the Asian F. velutipes in our study.

F. filiformis is one of the most important and popular edible mushrooms available commercially in China. It has been widely cultivated and consumed in oriental countries owing to its high nutritional value and attractive taste. It was reported that China is currently the largest producer of $F$. filiformis, with an annual production of 2.4 million tons [3]. F. filiformis also possesses tremendous pharmaceutical value and many bioactive constituents have been identified such as polysaccharides [4, 5, 6], flavonoid [7], sesquiterpenes, glycosides, proteins, phenols and so on [8, 9, 10]. These compounds have been demonstrated have antitumor, anticancer and anti-atherosclerotic, thrombosis inhibition, anti-aging and antioxidant effects [11, 12]. In addition, as a typical white-rot fungus, F. filiformis can effective degrade lignin and produce alcohol dehydrogenase, and thus make them potential for bioethanol production [13].

In past decades, researches on the genus of Flammulina have been carried out on phylogenetic taxonomy [1, 14, 15], genetic diversity [16,17], nutritional and chemical constituents $[18,19]$, pharmacological bioactivity $[20,21]$ and artificial cultivation $[22,23,24]$. Most of studies showed that $F$. velutipes possesses relative high content of carbohydrates, protein, amino acids and low fats or lipids, thus it generally was recognized as low energy delicacy [25]. In addition, bioactive polysaccharides (e.g. glucans and heteropolysaccharides), immunomodulatory protein (e.g. FIP-fve) and multiple bioactive sesquiterpenes were also isolated and identified from the extract, mycelium and fruiting bodies of $F$. velutipes [26]. Tang et al. [12] reviewed the compounds derived from the $F$. velutipes and their divers biological activities. More and more studies on chemical compounds and biological activity of the mushroom supported that $F$. velutipes should be exploited as a great source for development of functional foods, nutraceuticals and even pharmaceutical drugs [27].

Development of "omic" technology provided the powerful tools to understand the biology of edible mushroom including effective utilization for cultivation substrate (lignocellulose) [28, 29], the mechanism of fruiting body's formation and development and adaption for environment such as high temperature or cold stress [30,31,32]. For example, genome sequencing of the cultivars of Fvelutipes from Korea and Japan revealed the high capacity for lignocellulose degradation [28, 33]. Transcriptomic and proteomic analysis of $F$. velutipes identified key genes associated with cold and light stress-fruiting body formation and fruiting body morphogenesis [34]. These researches provided important information for its breeding and commercial cultivation of $F$. velutipes.

Recent advances in genome sequencing have revealed millions of putative biosynthetic gene clusters (BGCs) are hidden in fungal genomes [35]. Genome mining efforts have also allowed us understanding the silenced or activated biosynthetic pathway in microbe combined with the development of bioinformatics software such as antiSMASH, SMURF and PRISM [36]. For instances, genome-wide investigation of 66 cosmopolitan strains of Aspergillus fumigatus revealed 5 general types of variation in secondary genes cluster [37]. Identification of the tricyclic diterpene antibiotic pleuromuyilin gene clusters in genome-scale increased antibiotic production in Clitopilus passeckerianus [38]; Prediction of gene clusters involved in biosynthesis of terperoid/PKS in medicinal Hericium erinaceus by genome and transcriptome sequencing discovered a new family of diterpene cyclases in fungi $[39,40]$ and identification of the candidate cytochromes $\mathrm{P} 450$ s genes cluster possible related to triterpenoid biosynthesis in medicinal mushroom Ganoderma lucidum by genome sequencing improved the production of the medicinal effective compounds [41, 42].

However, as a popular edible mushroom that have wide spectrum of interesting biological activities, little is known for synthesis and regulation of bioactive secondary metabolites of $F$. filiformis. In previous experiments, we collected a wild strain of $F$. filiformis Liu355 from Longling, Yunnan and demonstrated that it could tolerate relatively high temperature during fruiting body formation (at $18{ }^{\circ} \mathrm{C}-22^{\circ} \mathrm{C}$ ) in the laboratory and it is prior to the commercial strains of $F$. filiformis (Asian commercial F. velutipes usually produce fruiting body under low temperature $\leq 15^{\circ} \mathrm{C}$ ) [16]. Thus, the wild strain is a potential and important genetic material for future breeding engineer because it can save large amounts of energy. Most interestingly, the chemical composition of the wild strain was different from other commercially cultivated strains of $F$. filiformis with more unique chemical compounds. Total 13 new sesquiterpenes with nor-eudesmane, spiroaxane, cadinane, and cuparane skeletons were isolated and identified from the wild strain Liu355 [9].

Thus, the aims of our study are to explore the genetic feature of this interesting wild strain of $F$. filiformis in genomic scale, to identify gene clusters associated with biosynthesis of bioactive secondary metabolites and to profile these candidate genes expression difference during the development of $F$. filiformis. This 
research will facilitate our understanding for its biology of the wild strain and provide a useful datasets for molecular breeding, as well for improving compounds production and producing novel compounds by next heterologous pathway and metabolic engineering in future.

\section{Results And Discussion}

General features of the F. filiformis genome

Prior to our study, three "genomes" assigned toF.velutipes" were publically available including the relative complete genome of the KACC42780 from Korea, L11 from China and a draft genome of the TR 19 from Japan. In this study, we sequenced the genome of wild strain of $F$. filiformis by small fragments library construction and did a comparative genome analysis on secondary metabolism gene clusters. The assembled genomes of the wild $F$. filiformis were $35.01 \mathrm{Mbp}$ with approximately 118-fold genome coverage. Total 10396 gene models were predicted, with an average sequence length of $1445 \mathrm{bp}$. The genome size and the number of predicted protein encoding genes are very similar to the public reference genome of $F$. filiformis (= Asian $F$. velutipes) (Table 1 ). Functional annotation of the predicted genes showed that more than half of predicted genes were annotated in NR database (6383 genes) and 1972,2582 , 837, 5794 genes were annotated in database SwissProt, KEGG, COG and GO, respectively. We identified 107 cytochrome P450 family genes, 674 genes encoding secretory proteins and 287 genes in CAZy database.

There have 17293 pan-genes among four strains of $F$. filiformis and pan-genome core comprised 4074 genes (on average $23.5 \%$ of each genome) (Fig. $1 \mathrm{~A}$ ). The proportion (23.5\%) of core genes in pan-genome analysis is similar to pan-genome analysis of 23 Corallococcus spp. [43]. But possibly, the number was lower than the actual number because that these genomes were not sequenced to completion. 3104 out of orthologous genes was annotated in KEGG database, 2722 genes have annotations in the GO database and 1055 genes are specific to the wild strain Liu355.

Functional characteristics of F. filiformis predicted genes

A KEGG enrichment analysis was performed to determine the functions of predicted genes of $F$. filiformis. The result showed that the highest number of genes of $F$. filiformis is involved in genetic information processing and translation ( 253 genes), followed by metabolism (carbohydrate metabolism with 243 genes). 21 genes were found participate into terpenoid and polyketides biosynthesis (Additional file 1: Figure S1).

Transcriptomic analysis and gene expression

We studied the gene expression differences across different development stages of $F$. filiformis including the monokaryotic (MK), dikaryotic mycelium (DK), primordium (PD) and fruiting body (FB) in transcriptomic level. Meanwhile, the DK of the cultivar strain of $F$. filiformis (CGMCC 5.642) also was transcriptomic sequenced. Three biological replicates were designed for each sample. An average clean data for each sample is 8.07-9.32 G. We mapped the clean reads to genomeof $F$. filiformis Liu 355 using the HISAT software and get relative high total mapping rate (92.63\%). In addition, the expression variation between samples was the smallest between MK and DK and was the greatest between the MK and FB of F. filiformis (Additional file 1: Figure S2).

Among 10396 gene models of $F$. filiformis, 9931 genes models were expressed (FPKM>5) across the four different tissues (MK, DK, PD and FB) of the wild strain and the mycelium of a cultivar strain of $F$. filiformis. 6577 genes were commonly expressed in all tissues and 151 genes were specific expressed in the cultivar strain, and 152,116,46, 199 genes were specific expressed in MK, DK, PD and FB of the wild strain of $F$. filiformis, respectively (Fig. 1B). Tissue-specific and high expression transcripts in F. filiformis Liu 355 were listed in Additional file 2: Table S1. Two new genes encoding ornithine decarboxylase (involved in ployamine synthesis) are highly expressed in mycelium of cultivar strains (Novel01369, Novel01744) and genes encoding oxidoreductase also has the highest expressed level (gene 830, FPKM>1000). Genes encoding agroclavine dehydrogenase, acetylxylan exterase, beta-glucan synthesis-associated protein and arabinogalactan endo-1,4-beta-galactosidase protein are significantly high expressed in fruiting bodies (FB) of the wild strain F. filiformis with more than 20100 fold change compared to mycelium. It was known that agroclavine dehydrogenase was involved in the biosynthesis of fungal ergot alkaloid ergovaline [44]; and beta-glucan synthesis-associated protein is probably linked the polysaccharide biosynthesis of fungal cell wall. Highly expression of these genes indicated that it probably play an important roles in fruiting bodies development and compounds enrichment.

Total 5131 genes (51.67\%) were up- or down regulated at least in one stage transitions such as from mycelium to primordium (PD vs DK, 3889 genes) and from primordium to fruiting body (FB vs PD, 3308 genes) (Fig. 1C). During primordial formation stage, 1780 genes are up-regulated and most of the genes belong to oxidoreductase activity (Go:0016491), hydrolase activity (Go: 0004553) and carbohydrate metabolism (Go: 0005975), down-regulated genes mainly was enriched with transmebrane transport (Go:0055085). During fruiting body development stage, genes related to fungal-type cell wall (Go:0009277) and structural constituent of cell wall (Go:0005199) are up regulated, reflecting the dramatic changes of cell wall structure during the development process. In addition, Go term enrichment of differentially expressed genes (DEGs) between wild strain Liu355 and cultivar strain CGMCC 5.642 showed that most genes displayed a similar expression profile, but peptide biosynthetic and metabolic process (Go: 0006518; Go:0043043), amide biosynthetic process (Go: 0043604) and ribonucleoprotein complex (Go: 1901566) are up-regulated in cultivar strain of CGMCC 5.642.

KEGG enrichment analysis showed that DEGs involved in glutathione metabolism is significantly enriched in dikayontic mycelium of wild strain Liu 355 compared to cultivar strain (Fig. 2). Thirty-three DEGs, including genes encoding glutathione S-transferase, ribonucleoside-diphosphate reductase, 6phosphogluconate dehydrogenase, cytosolic non-specific dipeptidase, gamma-glutamyltranspeptidase, glutathione peroxidase etc. participated in this pathway. Study on the glutathione metabolism of the filamentous fungus Aspergillus nidulans indicated thatglutathione itself and glutathione metabolic enzymes play a crucially important role in the germination of conidiospores, as well as remarkable contribute to the general stress tolerance of fungi [45]. High expression of genes related to glutathione metabolism in wild strain of $F$. filiformis implied the strain probably has strong environment adaptation and is potential better breeding resource. In addition, during the primordial and fruiting body development stage, MAPK signaling pathway (45 DEGs) and starch and 
sucrose metabolism (26 DEGs) is significantly enriched. Tyrosine metabolism, biosynthesis of secondary metabolites and glycosphingolipid biosynthesis are also significantly enriched in fruiting bodies formation stage.

\section{Genes involved polysaccharide biosynthesis of F. filiformis}

Polysaccharides (PSs) are an important and bioactive component of $F$. filiformis and other edible and medicinal mushrooms [46]. Glucan is well studied PSs and can be produced by different fungi, such as lentinan, ganoderan, grifolan, and schizophyllan [47]. Previous studies showed that

F. velutipes polysaccharide (FVP) have good immunomodulation activities, anti-proliferation and improved the learning and memory impairment in rats [4, 5]. Recently, an interesting result showed that FVP even could be used as a material of edible film for packaging of food and vegetables because of their excellent features of film-forming and edibility safety [48].

The biosynthetic pathways of polysaccharide (PSs) involved synthesis of nucleotide sugar precursors, assembly of repeating monosaccharide units, and the polymerization process [42]. Although the exactly biosynthetic pathways of mushroom PSs remain unclear, polysaccharide synthesis is not a template-driven process, but instead the structures are determined primarily by the complement of polysaccharide-modifying enzymes present in any organism [49]. Phosphoglucomutase (PGM) and UDP-glucose pyrophosphorylase (UGP) are known as important enzymes in the biosynthetic pathway of nucleotide sugar precursors [46]. In addition, based on identification of intermediate compounds and activities of synthesis-related enzymes, researchers constructed a simplified biosynthetic pathway of mushroom PSs and inferred several enzymes such as glucokinase (GK), phosphoglucose isomerase (PGI), fructose-1,6biphosphatase (FBPase) and UDP-Glc pyrophosphorylase (UGP) probably involved in the biosynthetic pathways of PSs of edible mushroom [42, 46]. These enzymes play key roles in glycolysis and gluconeogenesis pathway and starch and carbohydrate metabolism.

We identified total 80 genes related to PSs biosynthesis involved glycolysis and gluconeogenesis in KEGG pathway (KEGG map 00010) [50] at genome level, including Glucose-6-phosphate isomerase (GPI), Fructose-1,6-bisphosphatase (FBP), Mannose-6-phosphate isomerase (PMI). The expression profile of these genes showed that genes encoding Zinc-type alcohol dehydrogenase are both up-regulated in mycelium of wild strains compared to cultivar strain and in fruiting body stage compared to mycelium of wild strain of $F$. filiformis (Additional file 1: Figure S3 and Additional file 2: Table S2). Genes encoding glycerol 2-dehydrogenase (gene9557, gene2028), 7-bisphosphatase (gene 2929), alcohol dehydrogenase (gene7891-D2, gene 9773-D2) and aryl-alcohol dehydrogenase (gene 4871, gene 612) are up-regulated in mycelium of the wild strain. Gene encoding mannose-1-phosphate guanylyltransferase (gene 11132-D3) have highest expression level in mycelium of wild strain with more than 200-fold change compared to cultivar strain. Genes encoding glycerol 2dehydrogenase (gene 894) and sugar phosphatase are up-regulated expression in fruiting bodies stage of wild strain.

Moreover, based on predicted metabolic enzymes related toPSs biosynthesis of Ganoderma lucidum [46], we identified 21 putative essential enzyme involved in PSs biosynthesis in F. filiformis using protein sequencing homology search method (Table 2), including glucose-6-phosphate isomerase, hexokinase, mannose-6-phosphate isomerase, UDP-glucose dehydrogenase, galactokinase and transketolase etc. Among them, genes encoding UDP-glucose pyrophosphorylase, UDP-glucose dehydrogenase and fructose-bisphosphate aldolase have relative high transcripts in all samples analyzed (FPKM $>100$ ). These candidate genes will be functional verification by experiment in future, combined the quantification of PSs in different tissue.

\section{Carbohydrate active enzymes (CAZymes)}

Secreted carbohydrate-degrading enzymes are crucial enzymes for fungal biology, both for fungal cell wall turnover and for degradation of external carbohydrate sources. They are also responsible for the biosynthesis, degradation and modification of oligo- and polysaccharides as well as of glycoconjugates [51]. We performed sequencing similarity search against the CAZy database using Blastx $\left(E<10^{-5}\right)$ and totally identified 287 carbohydrateactive related genes in F. filiformis genome, including 149 Glycoside-Hydrolases (GH), 48 Auxiliary Activities (AA) containing redox enzymes, 36 glycosyltransferases (GT), 29 Carbohydrate-binding module (CBM), 13 Carbohydrate Esterases (CE) and 12 Polysaccharide Lyases (PL). The number of secreted carbohydrate-degrading enzymes (mostly GHs) is nearly four times than polysaccharide biosynthetic enzyme (GT), indicating their dependence on external carbohydrate sources for growth and development as wood-rot fungi. Genes encoding beta-1,3-glucan-binding protein (GH16) are most abundant with 23 members in F. filiformis genome. Previous studies showed that GH16 enzymes are involved in the formation of the chitin-b-1,3-glucan complex, suggesting a role in the processing of fungal cell-wall polysaccharides [51]. The expression profile of these genes related to CAZys is diverse (Fig. 3). Seven genes of $23 \mathrm{GH} 16$ family are up-regulated expression in DK mycelium stage compared to MK (three genes annotated as candidate glycosidase and four genes as beta-glucan synthesis-associated protein), four genes of $\mathrm{GH} 16$ family are up-regulated in fruiting bodies compared to primordium.

Besides GH16, seven out of the 14 GH5 members (four genes annotated as Glucan 1,3-beta-glucosidase, two genes annotated as endoglucanase and another annotated as mannan endo-1,4-beta manosidase) are differentially up-regulated in DK mycelium of the wild strain compared to MK mycelium and five genes belong to $\mathrm{GH} 5$ family are up-regulated in primordium stage compared to mycelium. Eight members out of $11 \mathrm{GH} 43$ are up-regulated in primordium compared to mycelium.

\section{Predicted bioactive secondary metabolism genes clusters of F. filiformis}

Besides the macronutrients and micronutrients present in the $F$. filiformis, a large number of structurally diverse bioactive secondary metabolites, especially various novel sesquiterpenes and norsequiterpenes were identified from the mycelium and fruiting bodies of $F$. filiformis [9]. The genes involved in sesquiterpenes biosynthesis were described previously [9]. In this study, the gene cluster encoding terpenoid and PKS were re-examined using the genome and transcriptome resource. In total, 12 gene clusters related to terpenoid biosynthesis and two gene clusters for polyketide biosynthesis were predicted (Fig. 4 , Additional file 2: Table S3). Compared with other three cultivar strains (KACC42780, TR 19 and L11 with genome sequencing), the numbers of gene clusters involved in terpene, PKS, NRPS and Siderophore biosynthesis are different and the gene number related to terpene synthesis is higher in wild strain Liu355 (119 genes) than cultivar strain L11 (81 genes) in our study (Table 3). 
119 genes of 12 terpenoid clusters divided into 10 clades according to their expression level (Additional file 1: Figure S4). Most genes in clade II are upregulated in primodium stage of wild strain Liu 355 compared to mycelium stage, including encoding 4,5-DOPA dioxygenase extradiol-like protein (gene 3103) and squalene synthase (gene 3428) involved in biosynthesis of squalene, a precursor of terpenoid compounds. Genes in VIII-X clades are significantly differently expressed in mycelium of wild strain Liu 355 compared to cultivar strain and in the fruiting body stage compared with mycelium of wild strain Liu 355, including key enzymes involved in terpenoid biosynthesis such as protoilludene synthase, candidate peroxisomal acyl-coenzyme A oxidase, L-amino acid amidase and cytochorme P450. It probably explained more divers bioactive compound in wild stains than cultivar strain in previous study.

\section{Putative genes for sesquiterpenoid biosynthesis in F. filiformis}

Sesquiterpene compounds are mainly bioactive secondary metabolites in Flammulina. Chemistry investigation of six strains of $F$. filiformis in previous reports revealed that the wild strain Liu355 contained many new sesquiterpens with various skeletons, including cuparene-type and sterpurane type sesquiterpenes [9]. However, little is known about sesquiterpene synthases (STSs) in Basidiomycota until the mostly recent years, although these fungi can produce divers bioactive sesquiterpenes. Thanks to the identification of sesquiterpene synthases from Coprinus cinereus (Cop1-6) and Omphalotus olearius, it provided a useful guidance for subsequent development of in silico approaches for the directed discovery of new sesquiterpene synthases and their associated biosynthetic genes [52]. In our study, we performed a genome-scale homologous search with sesquiterpene synthases of $O$. olearius, $C$. cinereus and Hericium erinaceus, 12 homologue sequences with considerable similarity (e-value $<10^{-5}$ ) to the known biochemically characterized sesquiterpene synthases were identified in the genome of F. filiformis. The phylogenetic analysis showed that these genes consist of four clades (Additional file 1: Figure S5, Additional file 2: Table S4). Eight genes out of 12 genes related to sesquiterpene biosynthesis have closely phylogenetic relationship with genes identified from cultivar Flammulina in the previous study [9]. 12 STSs genes of F. filiformis include five genes encoding delta (6)-protoilludene synthase (gene1663-D2, gene9115, gene2784 and gene9115-D2, gene 6325-D2), two genes encoding trichodiene synthase (gene1140, gene 2254), two genes encoding alpha-muurolene synthase (gene1358-D2, gene1358), and one gene encoding glucose-6-phosphate isomerase. Among them, genes encoding delta (6)-protoilludene synthase are upregulated in fruiting body stage compared to mycelium and also up regulated in DK mycelium compared to MK of the wild strain Liu355 (Table 4).

\section{Putative genes for polyketides biosynthesis in F. filiformis}

Polyketides are major group of secondary metabolites isolated from bacteria and fungi and fungi in particular have been important sources of natural product polyketide pharmaceuticals. Structural complexity of these polyketides makes them interesting and useful bioactive compounds and these same features also make them difficult and expensive to prepare and scale-up using synthetic methods [53]. In past, understanding of the biosynthesis and regulation of polyketide in fungi are extremely difficult because of lacking of established genetic tools. Recent researches illustrated that Saccharomyces cerevisiae have potential as a tool for mining, studying and engineering fungal polyketide synthases during to their many advantages benefits such as unicellular organism well suited for large scale fermentation, limited native secondary metabolism and a number of developed genetic tools for protein expression and pathway constriction [53].

The diverse structures of polyketides are biosynthesized from short-chain carboxylic acid units by polyketide synthases (PKSs), PKSs have been classified into type I, type II and type III based on their product profiles and catalytic domain architecture [54]. By gene clusters prediction using the antiSMASH, we found 30 genes of two gene clusters responsible for Type I PKS. They mainly located in a single scaffold 24 and 78 in F. filiformis genome, respectively (Fig. 4 , Additional file 2: Table S3). The two gene clusters both included core genes encoding polyketide synthase (gene 8217 and gene 1373 ). Genes located scafflold 78 are most up-regulated in wild strain mycelium compared with cultivar strain, including erythronolide synthase (gene 1374), polyketide synthase (gene1373), benzoate 4-monooxygenase (gene 1372), indicating that polypeptide compounds are probably abundant in mycelium of this mushroom and especially in wild strain.

\section{Cytochrome P450s in F. filiformis genome}

It is reported that a more divergent cytochrome P450 oxidase could be involved in secondary biosynthesis [55]. So, we searched the genome of the $F$. filiformis for proteins with a P450 conserved domain using NCBI's CDD tool and BLASTp [39]. We yielded 107 genes in cytochrome P450 family, including nine putative trichodiene oxygenase, 31 0-methylsterigmatocystin oxidoreductase, five benzoate 4-monooxygenase, two linoleate 10R-lipoxygenase, two ent-kaurene oxidase, lanosterol 14-alpha and flavonoid hydroxylase and other candidate cytochrome P450. 102 genes out of them have diverse expression profile across different tissue of F. filiformis (Additional file 1: Figure S4). 26 CYP450 genes are up-regulated in mycelium of wild strain compared to cultivar strain and the gene 54280-D3 have most high expression level with more than 500 fold change. 21 CYP450 genes are up-regulated in fruiting bodies stage compared to mycelium and gene encoding benzoate 4-monooxygenase have highest transcript level with 15 fold change. In primodim formation stage, gene encoding docosahexaenoic acid omega-hydroxylase is highest differential expressed.

\section{Heat shock protein correspond to temperature change in F. filiformis}

Besides with unique compounds, the wild strain of $F$. filiformis Liu 355 can produce the fruiting body at relative high temperature than current commercial strains, implying it is a potential excellent breeding resource. A temperature downshift (cold stimulation) is considered to be one of the most important and essential environmental factors for the fruiting initiation and fruiting body formation of $F$. velutipes [34]. In generally, mycelia of $F$. velutipes can grow vegetative at $20-24{ }^{\circ} \mathrm{C}$ and fruiting at an optimum temperature for $12-15^{\circ} \mathrm{C}$ [32]. In our study, the wild strain of Liu 355 can grow fruiting bodies at $18{ }^{\circ} \mathrm{C}$ at the laboratory. Therefore, it is potential excellent genetic material for $F$. velutipes breeding. Proteomic sequencing revealed that the expression of proteins related to energy metabolism (e. g. catalase, glucose-6-phosphate isomerase, trehalase and beta-glucosidase), amino acids biosynthesis (e. g. argininosuccinate synthase) and signal pathway (e.g. BAR adaptor protein) are dramatically increased after long-term cold stress [32, 34]. In addition, histidine kinases, response regulators, and sometimes histidine-containing phosphotransfer proteins were also reported play crucial roles in response to cold stress in cyanobacterium Synechocystis sp. and Arabidopsis [56, 57]. Transcriptomic sequencing revealed that histidine kinase and proteins involved in MAPK pathway 
(e.g. STE protein kinase, MAPK kinase kinase) and $\mathrm{Ca}^{2+}$ signal transduction pathway (calcium-dependent protein kinase) were differentially expressed in $F$. velutipes [31].

The heat shock protein (HSP) family was known that it positively correlated with the organism thermotolerance [58]. In this study, 28 genes annotated as HSP were identified in F. filiformis genome (Fig. 5). Among them, six genes are significantly up-regulated in wild strain Liu 355 compared to cultivar strain and encoding HSP12, HSPC4, HSP104, LHS1 and GRP78, respectively. HSP12 is part of a group of small heat shock proteins (HSP) that function as chaperone proteins and are ubiquitously involved in nascent protein folding by protecting proteins from misfolding and partially characterized as a stress response and expression of HSP12 protein was observed in response to cold stress [59]. In S. cerevisiae and C. albicans, HSP104 in association with HSP40 and HSP70 helps in reactivation and aggregation of denatured protein, by providing disaggregated protein to HSP40 and HSP70 as a substrate [60]. Expression of HSP104 and HSP70 is regulated by Hsf (heat-shock factor) interaction, which can be stimulated by heat stress in yeast [58]. However, the exactly molecular function of HSP in the high-temperature-tolerance of wild F. filiformis and adaptive mechanisms for relative high temperature need further study.

\section{Conclusion}

In our study, the genome and transcriptome sequencing, assembly and annotation of a high-temperature-tolerance of wild $F$. filiformis was carried out and the gene clusters associated with polysaccrides, terpenoid and polyketide biosynthesis were predicted. Comparative genomic analysis with other three Asian cultivars strains of F.velutipes ( = F. filiformis) revealed that the wild strain has a similar genome size and relative much more putative gene numbers related to secondary metabolites biosynthesis. Most of genes related terpeniod biosynthesis are up regulated in primodium and fruiting body of the wild strain, while PKS genes are usually up-regulated in the mycelium of wild strain but the exactly regulation pathway is unresolved in this study.

Six genes belongs to the heat shock protein (HSP) family were significantly up-regulated in wild strain Liu 355 compared to cultivar strain, including HSP12, HSPC4, HSP104, LHS1 and GRP78, which is possible responsible for high-temperature-tolerance of wild F. filiformis developing fruiting body in relative high temperaturebut these genes expression in other different strain of $F$. filiformis, especially in strain with low-temperature development will be verified in next step.

Our study provides an important genetic dataset for the potential breeding materials of $F$. filiformis and put a foundation for better understanding the biology of F. filiformis.

\section{Methods}

\section{Culture of strains}

The wild strain Liu355 was isolated from the fruiting body of $F$. filiformis collected from Longling, Yunnan province, southwestern China and its ITS sequence was listed under GenBank accession number KP867925 [9]. The haploid monokaryotic strain F. filiformis Liu355 (deposited in mycological lab of Institute of Medicinal Plant Development, Chinese Academy of Medical Sciences) was prepared by protoplast mononuclear method and was grown on potato dextrose agar (PDA) at room temperature for 2-3 weeks in the dark (Additional file 1: Figure S6). The fruiting bodies were obtained in sterile plastic bottles containing on growth substrate (cotton seed hulls $78 \%$, wheat bran $20 \%, \mathrm{KH}_{2} \mathrm{PO}_{4} 0.1 \%, \mathrm{MgSO}_{4} 0.1 \%$, sucrose $1 \%$ and ground limestone $1 \%$, with a moisture content of $70 \%)$ at $25^{\circ} \mathrm{C}$ for $30 \mathrm{~d}$, followed by cold-stimulation at $18^{\circ} \mathrm{C}$ and $90 \%$ humidity until primordial occurred. Cultures were maintained at low temperature $\left(18^{\circ} \mathrm{C}\right.$ and $75 \%$ humidity) to allow full fruiting body development [61]. In addition, the genomic data of two cultivars strains from Korea (KACC42780, Bioproject PRJNA191921) and Japan (TR19, Bioproject PRJDB4587) were available from NCBI public database and genomic sequences of strain L11 (Bioproject PRJNA191865) was friendly provided by the Mycological Research Center, College of Life Sciences, Fujian Agriculture and Forestry University [62].

Genome, transcriptome sequencingand analysis

Total genomic DNA of $F$. filiformis was extracted from the mononuclear mycelium in the PDA medium using the Omega E. Z. N. A. fungal DNA midi kit (Omega, USA) according to manufacturer's instructions. Total DNA obtained was detected by agarose gel electrophoresis and quantified by Qubit. The strain of $F$. filiformis was sequenced using 350 bp paired-end reads on an Illumina HiSeq 4000 by PE150 strategy. Library construction and sequencing was performed at the Beijing Novogene Bioinformatics Technology Co. Ltd (Beijing, China). After removing adapter and low quality sequence using FastQC, the high quality reads were mapped into the reference genome sequence of $F$. filiformis L11 (Bioproject PRJNA191865) using BWA software. Functional annotation of the predicted genes was performed using BLAST against Gene ontology (GO), Kyoto Encyclopedia of Genes and Genomes (KEGG), SwissProt and NCBI non-redundant (NR) protein database [39]. Pan-genome analysis was carried out using the standalone CD-HIT tool to cluster orthologous proteins [63].

For transcriptomic sequencing, total RNA was extracted using the RNAeasy Plant Mini Kit (Qiagen) according to the manufacturer's protocols. Four samples were prepared including monokaryotic mycelium (MK), dikaryotic mycelium (DK), primordium (PD) and fruiting bodies (FB) of the strains Liu355 and each sample had three biological replicates. All samples were subjected to RNA-Seq on the Illumina HiSeq2000 platform (Illumina, San Diego, CA, USA).

Bioinformatics analysis was done based on clean data with high quality and the RNA-seq reads were mapped to the $F$. filiformis genome (Liu355) using TopHat v2.0.1253 [64]. HTSeq v0.6.1 software was used to count the read numbers mapped to each gene [65]. The FPKM value was used to calculate gene expression, and the upper-quartile algorithm was used to correct the gene expression. Gene differential expression analysis was performed using the DESeq $\mathrm{R}$ package (1.18.0) using a corrected p-value [66]. Genes with an adjusted P-value $<0.05$ were assigned as differentially expressed. Hierarchical clustering of gene expression was conducted using Genesis 1.7.7[67].

Prediction of gene clusters

Page 6/16 
The biosynthetic gene clusters were predicted using antiSMASH 3.0 software [68]. The antiSMASH currently offers a broad collection of tools and databases for automated genome mining and comparative genomics for wide variety of different classes of secondary metabolites [69]. In addition, homology sequence search method (Blast) was also used for identification of the genes related to terpenoid biosynthesis. The sesquiterpene synthases were identified based on multiple sequence alignments and phylogenetic analyses developed by Schmidt-Dannert group [52].

\section{Abbreviations}

BGCs: Biosynthetic gene clusters; PKS: Polyketide synthase; KEGG: Kyoto Encyclopedia of Genes and Genomes; COG: Clusters Orthologous Groups; GO: Gene Ontology; CAZy: Carbohydrate-Active Enzymes; MK: monokaryotic; DK: dikaryotic; PD: primordium; FB: fruiting body; FPKM: Fragments Per Kilobase of transcript per Million fragments mapped; MAPK: mitogen-activated protein kinase; PSs: Polysaccharides; FVP: F. velutipes polysaccharide; PGM:

Phosphoglucomutase; UGP: UDP-glucose pyrophosphorylase; GK: glucokinase; PGI: phosphoglucose isomerase; GPI: Glucose-6-phosphate isomerase; FBP: Fructose-1,6-bisphosphatase; PMI: Mannose-6-phosphate isomerase; GH: Glycoside-Hydrolases; $A A$ : Auxiliary Activitie; $G T$ : glycosyltransferases; $C B M$ Carbohydrate-binding module; $C E$ : Carbohydrate Esterases; $P$ L: Polysaccharide Lyases; STSs: sesquiterpene synthases; $C D D$ : Conserved Domain Database; CYP: Cytochrome P450 proteins; HSP: The heat shock protein; Hsf: heat-shock factor; ITS: internal transcribed spacer; PDA: potato dextrose agar; DEGs: differentially expressed genes (DEGs).

\section{Declarations}

Acknowledgments

The authors are grateful to Prof. Francis Martin and Prof. HW Liu for critical discussion and suggestion.

Funding

This study was supported by National Key R \& D Program of China (2017YFC1701900), the Natural Science Foundation of China (81573527) and National Basic Research Program of China (2014CB138304).

Author's contributions

JC and SG designed the experiments. JL, YT, BL, XL, YL WX performed the experiment. ZY and $\mathrm{HL}$ collected the sample and provided the strain. BX sequenced and provided the reference genome of $F$. velutipes $\mathrm{L} 11$. JC, KM and XZ analyzed the data, JC contributed to text writing and literature search and SG reviewed and revised the manuscript.

Availability of data and materials

The datasets supporting the results of this article are included with in the article and additional files. The genomic and transcriptomic data have been deposited to GenBank database with the dataset identifier PRJNA531555 for genome and PRJNA530834 for tanscriptome.

Ethics approval and consent to participate

Not applicable.

Consent for publication

Not applicable.

Competing interests

The authors declare that they have no competing interests.

\section{References}

1. Ge ZW, Liu XB, Zhao K, Yang ZL. Species diversity of Flammulina in China: new varieties and a new record. Mycosystema. 2015;34: 589-603.

2. Wang PM, Liu, XB, Dai YC, Horak E, Steffen K, Yang ZL. Phylogeny and species delimitation of Flammulina: taxonomic status of winter mushroom in East Asia and a new European species identified using an integrated approach. Mycological Progress. 2018;17(9):1013-1030.

3. Li X, Li Y. Quality comparison and analysis on white Flammulina velutipes grown with bottle lines in China. Edible Fungi China. 2014;33: 20-24.

4. Lin L, Cui F, Zhang J, Gao X, Zhou M, Xu N, et al. Antioxidative and renoprotective effects of residue polysaccharides from Flammulina velutipes.Carbohydr Polym. 2016;46:388-395.

5. Su A, Yang W, Zhao L, Pei F, Yuan B, Zhong L, et al. Flammulina velutipes polysaccharides improve scopolamine-induced learning and memory impairment in mice by modulating gut microbiota composition. Food Funct. 2018;9(3):1424-1432.

6. Zhang T, Ye J, Xue C, Wang Y, Liao W, Mao L, et al. Structural characteristics and bioactive properties of a novel polysaccharide from Flammulina velutipes. Carbohydr Polym. 2018;197:147-156.

7. Hu Q, Yu J, Yang W, Kimatu BM, Fang Y, Ma N, et al. Identification of flavonoids from Flammulina velutipes and its neuroprotective effect on pheochromocytoma-12 cells. Food Chem. 2016; 204:274-282. 
8. Wang Y, Bao L, Yang X, Li L, Li S, Gao H, et al. Bioactive sesquiterpenoids from the solid culture of the edible mushroom Flammulina velutipes growing on cooked rice. Food Chem. 2012;132(3):1346-1353.

9. Tao Q, Ma K, Yang Y, Wang K, Chen B, Huang Y, et al. Bioactive sesquiterpenes from the edible mushroom Flammulina velutipes and their biosynthetic pathway confirmed by genome analysis and chemical evidence. J Org Chem. 2016;21: 81(20): 9867-9877.

10. Li HP, Yang WJ, Qu SX, Pei F, Luo X, Mariga AM, et al. Variation of volatile terpenes in the edible fungi mycelia Flammulina velutipes and communications in fungus-mite interactions. Food Res Int. 2018;103:150-155.

11. Rahman MA, Abdullah N, Aminudin N. Antioxidative effects and inhibition of human low density lipoprotein oxidation in vitro of polyphenolic compounds in Flammulina velutipes (Golden Needle Mushroom). Oxid Med Cell Longev. 2015;403023.

12. Tang C, Hoo PC, Tan LT, Pusparajah P, Khan TM, Lee LH, et al. Golden needle mushroom: A culinary medicine with evidenced-based biological activities and health promoting properties. Front Pharmacol. 2016;7: 474.

13. Kasprzycka A, Lalak-Kańczugowska J, Tys J. Flammulina velutipes treatment of non-sterile tall wheat grass for enhancing biodegradability and methane production. Bioresour Technol. 2018;263:660-664.

14. Avin FA, Bhassu S, Shin TY, Sabaratnam V. Molecular classification and phylogenetic relationships of selected edible Basidiomycetes species. Mol Biol Rep. 2012;39(7):7355-7364.

15. Wang Y, Bao L, Liu D, Yang X, Li S, Gao H, et al. Two new sesquiterpenes and six norsesquiterpenes from the solid culture of the edible mushroom Flammulina velutipes. Tetrahedron. 2012;68(14):3012-3018.

16. Liu XB, Feng B, Li J, Yan C, Yang ZL. Genetic diversity and breeding history of winter mushroom (Flammulina velutipes) in China uncovered by genomic SSR markers. Gene. 2016; 591: 227-235.

17. Wang Q, Zhang J, Li C, Wang B, Nong W, Bian Y, et al. Phenotypic and genetic diversity of the culinary-medicinal winter mushroom Flammulina velutipes (Agaricomycetes) in China. Int J Med Mushrooms. 2018; 20(6):517-536.

18. Reis FS, Barros L, Martins A, Ferreira IC. Chemical composition and nutritional value of the most widely appreciated cultivated mushrooms: an interspecies comparative study. Food Chem Toxicol. 2012;50(2):191-197.

19. Tsai SY, Huang EW, Lin CP. Compositional differences of the winter culinary-medicinal mushroom, Flammulina velutipes (Agaricomycetes), under three types of light conditions. Int. J. Med. Mushrooms. 2017;19(3): 267-276.

20. Miyazawa N, Yoshimoto H, Kurihara S, Hamaya T, Eguchi F. Improvement of diet-induced obesity by ingestion of mushroom chitosan prepared from Flammulina velutipes. J Oleo Sci. 2018;67(2): 245-254.

21. Wu M, Luo X, Xu X, Wei W, Yu M, Jing N, et al. Antioxidant and immunomodulatory activities of a polysaccharide from Flammulina velutipes. J Tradit Chin Med. 2014;34(6):733-740.

22. Huang Q, Jia Y, Wan Y, Li H, Jiang R. Market survey and risk assessment for trace metals in edible fungi and the substrate role in accumulation of heavy metals. J Food Sci. 2015;80(7):H1612-1618.

23. Rugolo M, Levin L, Lechner BE. Flammulina velutipes: An option for "alperujo" use. Rev Iberoam Micol. 2016;33(4):242-247.

24. Xie C, Gong W, Yan L, Zhu Z, Hu Z, Peng Y. Biodegradation of ramie stalk by Flammulina velutipes: mushroom production and substrate utilization. AMB Express. 2017;7: 171.

25. Kalač P. A review of chemical composition and nutritional value of wild-growing and cultivated mushrooms. J Sci Food Agric. 2013;93(2): 209-218.

26. Wang Y, Bao L, Yang XL, Dai HQ, Guo H, Yao XS, et al. Four new cuparene-type sesquiterpenes from Flammulina velutipes. Helvetica Chimica Acta. 2012;95: 261-267.

27. Tung CH, Lin CC, Tung CC, Chen SF, Sheu F, Lu TJ. Combination of on-line desalting and HPLC-UV-ESI-MS for simultaneous detection and identification of FIP-fve and flammutoxin in Flammulina velutipes. J Food Drug Anal. 2018;26(3):1045-1053.

28. Park YJ, Baek JH, Lee S, Kim C, Rhee H, Kim H, et al. Whole genome and global gene expression analyses of the model mushroom Flammulina velutipes reveal a high capacity for lignocellulose degradation. PLoS ONE. 2014; 9(4): e93560.

29. Park YJ, Jeong YU, Kong WS. Genome sequencing and carbohydrate-active enzyme (CAZyme) repertoire of the white rot fungus Flammulina elastica. Int $J$ Mol Sci. 2018;19(8). pii: E2379.

30. Song HY, Kim DH, Kim JM. Comparative transcriptome analysis of dikaryotic mycelia and mature fruiting bodies in the edible mushroom Lentinula edodes. Sci Rep. 2018;8(1): 8983.

31. Wu TH, Ye ZW, Guo LQ, Yang XQ, Lin JF. De novo transcriptome sequencing of Flammulina velutipes uncover candidate genes associated with coldinduced fruiting. Journal of Basic Microbiology. 2018;58:698-703.

32. Liu JY, Meng JL, Chang MC, Feng CP, Yuan LG. iTRAQ-based quantitative proteome revealed metabolic changes of Flammulina velutipes mycelia in response to cold stress. Journal of Proteomics. 2017;156:75-84.

33. Kurata A, Fukuta Y, Mori M, Kishimoto N, Shirasaka N. Draft genome sequence of the basidiomycetous fungus Flammulina velutipes TR19. Genome Announc. 2016; 4(3):e00505-16.

34. Liu JY, Chang MC, Meng JL, Feng CP, Wang Y. A Comparative proteome approach reveals metabolic changes associated with Flammulina velutipes mycelia in response to cold and light stress. J Agric Food Chem. 2018; 66(14):3716-3725.

35. Doroghazi JR, Albright J, Goering AW, Ju KS, Haines RR, Tchalukov KA, et al. A road map for natural product discovery based on large-scale genomics and metabolomics. Nat Chem Biol. 2014;10(11):963-968. 
36. Baral B, Akhgari A, Metsä-Ketelä M. Activation of microbial secondary metabolic pathways: Avenues and challenges. Synth Syst Biotechnol. 2018;3(3):163-178.

37. Lind AL, Wisecaver JH, Lameiras C, Wiemann P, Palmer JM, Keller NP, et al. Drivers of genetic diversity in secondary metabolic gene clusters within a fungal species. PLoS Biol. 2017;15(11): e2003583.

38. Bailey AM, Alberti F, Kilaru S, Collins CM, de Mattos-Shipley K, Hartley AJ, et al. Identification and manipulation of the pleuromutilin gene cluster from Clitopilus passeckerianus for increased rapid antibiotic production. Sci Rep. 2016;6: 25202.

39. Chen J, Zeng X, Yang YL, Xing YM, Zhang Q, Li JM, et al. Genomic and transcriptomic analyses reveal differential regulation of diverse terpenoid and polyketides secondary metabolites in Hericium erinaceus. Sci Rep. 2017;7:10151.

40. Yang YL, Zhang S, Ma K, Xu Y, Tao Q, Chen Y, et al. Discovery and characterization of a new family of diterpene cyclases in bacteria and fungi. Angew. Chem. Int. Ed. 2017; 56: $4749-4752$.

41. Chen S, Xu J, Liu C, Zhu Y, Nelson DR, Zhou S, et al. Genome sequence of the model medicinal mushroom Ganoderma lucidum. Nat Commun. 2012;3: 913.

42. Ma Z, Ye C, Deng W, Xu M, Wang Q, Liu G, et al. Reconstruction and analysis of a genome-scale metabolic model of Ganoderma lucidum for improved extracellular polysaccharide production. Front Microbiol. 2018;9:3076.

43. Livingstone PG, Morphew RM, Whitworth DE. Genome sequencing and pan-Genome analysis of 23 Corallococcus spp. strains reveal unexpected diversity, with particular plasticity of predatory gene sets. Front Microbiol. 2018; 199:3187.

44. Lorenz N, Wilson EV, Machado C, Schardl CL, Tudzynski P. Comparison of ergot alkaloid biosynthesis gene clusters in Claviceps species indicates loss of late pathway steps in evolution of C. fusiformis." Appl Environ Microbiol. 2007;73:7185-7191.

45. Bakti F, Király A, Orosz E, Miskei M., Emri T, Leiter É, Pócsi I. Study on the glutathione metabolism of the filamentous fungus Aspergillus nidulans. Acta Microbiol Immunol Hung. 2017; 64(3): 255-272.

46. Ruthes AC, Smiderle FR, lacomini M. Mushroom heteropolysaccharides: A review on their sources, structure and biological effects. Carbohydr Polym. 2016;136:358-375.

47. Wang Q, Wang F, Xu, Z, Ding Z. Bioactive mushroom polysaccharides: a review on monosaccharide composition, biosynthesis and regulation. Molecules. 2017;22(6):pii: E955.

48. Du H, Hu Q, Yang W, Pei F, Kimatu BM, Ma N, Fang Y, Cao C, Zhao L. Development, physiochemical characterization and forming mechanism of Flammulina velutipes polysaccharide-based edible films. Carbohydrate Polymers. 2016;152: 214-221.

49. Zeidan AA, Poulsen VK, Janzen T, Buldo P, Derkx PMF, Øregaard G, Neves AR. Polysaccharide production by lactic acid bacteria: from genes to industrial applications. FEMS Microbiol Rev. 2017;41(Supp_1): S168-S200.

50. Kanehisa M, Tanabe M, Sato Y, Morishima K. KEGG: new perspectives on genomes, pathways, diseases and drugs. Nucleic Acids Res. 2017;45, D353D361.

51. Veneault-Fourrey C, Commun C, Kohler A, Morin E, Balestrini R, Plett J, et al. Genomic and transcriptomic analysis of Laccaria bicolor CAZome reveals insights into polysaccharides remodeling during symbiosis establishment. Fungal Genet Biol. 2014;72:168-181.

52. Wawrzyn GT, Quin MB, Choudhary S, López-Gallego F, Schmidt-Dannert C. Draft genome of Omphalotus olearius provides a predictive framework for sesquiterpenoid natural product biosynthesis in Basidiomycota. Chem Biol. 2012;19:772-783.

53. Bond C, Tang Y, Li L. Saccharomyces cerevisiae as a tool for mining, studying and engineering fungal polyketide synthases. Fungal Genet Biol. 2016; 89 : $52-61$.

54. Shen B. Polyketide biosynthesis beyond the type I, II and III polyketide synthase paradigms. Curr Opin Chem Biol. 2003;7: 285-295.

55. Shin J, Kim JE, Lee YW, Son H. Fungal Cytochrome P450s and the P450 Complement (CYPome) of Fusarium graminearum. Toxins (Basel). 2018;10(3): pii: E112.

56. Shimura Y, Shiraiwa Y, Suzuki I. Characterization of the subdomains in the N-terminal region of histidine kinase Hik33 in the cyanobacterium Synechocystis sp. PCC 6803. Plant Cell Physiol. 2012; 53:1255-1266.

57. Jeon J, Kim NY, Kim S, Kang NY. A subset of cytokinin two component signaling system plays a role in cold temperature stress response in Arabidopsis. J Biol Chem. 2010; 285: 23369-23384.

58. Shui W, Xiong Y, Xiao W, Qi X, Zhang Y, Lin Y, Guo Y, Zhang Z, Wang Q, Ma Y. Understanding the mechanism of thermotolerance distinct from heat shock response through proteomic analysis of industrial strains of Saccharomyces cerevisiae. Mol Cell Proteomics. 2015;14: A779-A780.

59. Tiwari S, Thakur R, Shankar J. Role of heat-shock proteins in cellular function and in the biology of fungi. Biotechnol. Res Int. 2015;132635.

60. Glover JR, Lindquist S. “Hsp104, Hsp70, and Hsp40: a novel chaperone system that rescues previously aggregated proteins,” Cell.1998; 94(1): 73-82.

61. Luo R, Guo L, Lin J, Han F, Li Q, Kang L. A Novel high-temperature-tolerant Strain of Flammulina velutipes by mutagenesis. Edible Fungi of China. 2016;35(4):18-23.

62. Liu F. Preliminary study of Flammulina velutipes genome and transcriptome. PhD Dissertation, Fujian Agriculture and Forestry University, Fuzhou. 2014;1-117 (in Chinese).

63. Fu L, Niu B, Zhu Z, Wu S, Li W. CD-HIT: accelerated for clustering the next-generation sequencing data. Bioinformatics. 2012;28: 3150-3152.

64. Kim D, Pertea G, Trapnell C, Pimentel H, Kelley R, Salzberg S L. TopHat2: accurate alignment of transcriptomes in the presence of insertions, deletions and gene fusions. Genome Biol. 2013;14(4):R36.

65. Anders S, Pyl PT, Huber W. HTSeq-a Python framework to work with high-throughput sequencing data. Bioinformatics. 2015;31(2):166-169. 
66. Wang Z, Gerstein M, Snyder M. RNA-Seq: a revolutionary tool for transcriptomics. Nat Rev Genet. 2009;14(1):57-63.

67. Sturn A, Quackenbush J, Trajanoski Z. Genesis: cluster analysis of microarray data. Bioinformatics. 2002;18: $207-208$.

68. Weber T, Blin K, Duddela S, Krug D, Kim HU, Bruccoleri R, et al. antiSMASH 3.0-a comprehensive resource for the genome mining of biosynthetic gene clusters. Nucleic Acids Res. 2015;43:W237-243.

69. Blin K, Kim HU, Medema MH, Webe T. Recent development of antiSMASH and other computational approaches to mine secondary metabolite biosynthetic gene clusters. Brief Bioinform. 2017;1-11.

\section{Tables}

Table 1. Genomic features of four strains of Flammulina filiformis (=Asian F. velutipes).

\begin{tabular}{lllll} 
Strain voucher & Liu355 & L11 & TR19 & KACC42780 \\
\hline Accession number & PRJNA531555 & PRJNA191865 & PRJNA191921 & PRJDB4587 \\
\hline strain original & Wild, Yunnan, China & Clutivated, Fujian, China & Cultivated, Japan & Cultivated, Korea \\
\hline Genome size $(\mathrm{Mb})$ & 35.01 & 34.33 & 34.79 & 35.64 \\
\hline Genome Coverage & $118 \times$ & & $132 \times$ & $37.2 \times$ \\
\hline No. of Scaffolds & 2040 & 1858 & 5130 & 11 \\
\hline No. of Contigs & 2060 & 28590 & 6405 & 500 \\
\hline Genes number & 10396 & 11526 & 10096 & 11038 \\
\hline Gene total length (bp) & $15027318(42.92 \%)$ & $17020883(49.58 \%)$ & $14905273(42.84 \%)$ & $15924075(44.68 \%)$ \\
\hline Gene average length & 1445 & 1477 & 1476 & 1443 \\
\hline G+C content(\%) & 52.31 & 52.46 & 52.35 & 52.31 \\
\hline P450 & 107 & 144 & - & - \\
\hline CAZy & 270 & 315 & - & 392 \\
\hline Secretory Protein & 674 & - & 245 & - \\
\hline Transposon pre number & 204 & 215 & - & 285
\end{tabular}

Due to technical limitations, table 2 is only available as a download in the supplemental files section

Table 3. Putative genes and gene clusters related to secondary metabolism biosynthesis for the F. filiformis

\begin{tabular}{|c|c|c|c|c|c|c|c|c|}
\hline Strains & Liu 355 (wild stra & & L11(cultivar) & & KACC42780(Kore & & TR19(Japan) & \\
\hline Clusters & Clusters_number & $\begin{array}{l}\text { gene } \\
\text { number }\end{array}$ & Clusters_number & $\begin{array}{l}\text { gene } \\
\text { number }\end{array}$ & Clusters_number & $\begin{array}{l}\text { gene } \\
\text { number }\end{array}$ & Clusters_number & $\begin{array}{l}\text { gene } \\
\text { number }\end{array}$ \\
\hline Siderophore & 2 & 5 & 1 & 5 & 1 & 15 & 1 & 4 \\
\hline NRPS & 1 & 17 & 1 & 15 & 1 & 44 & 1 & 13 \\
\hline Terpene & 12 & 119 & 10 & 81 & 17 & 139 & 15 & 115 \\
\hline typelPKS & 2 & 32 & 1 & 18 & 2 & 34 & 2 & 30 \\
\hline cf_putative & 6 & 71 & 3 & 53 & 52 & 1286 & 39 & 696 \\
\hline
\end{tabular}

Table 4. Expression level of 10 genes encoding enzymes involved into sesquiterpenoid biosynthesis in F. filiformis

\#FPKM value is the mean expression value of three biological replicates

\#Abbreviation: MK: monokaryontic mycelium; DK: Dikaryontic mycelium; FB: fruiting body; PD: primordia

\#black bont means genes significantly up-regulated;

\#Note: among 12 genes, the expression of 2 genes (gene1358;gene2785) have't been detected in varous tissues. 


\begin{tabular}{|c|c|c|c|c|c|c|c|c|}
\hline Gene ID & chromosome & length & wild_FB_FPKM & wild_primordia_FPKM & wild_MK_FPKM & wild_DK_FPKM & clutivar_DK_FPKM & $\begin{array}{l}\text { log2ratio } \\
\text { wild_DK/cu } \\
\text { DK) }\end{array}$ \\
\hline $\begin{array}{l}\text { gene1358- } \\
\text { D2 }\end{array}$ & Scaffold 262 & 1005 & 45.38 & 48.94 & 124.54 & 82.53 & 38.31 & 0.86 \\
\hline gene3100 & Scaffold10 & 2559 & 66.43 & 69.62 & 104.75 & 95.85 & 80.55 & 0.01 \\
\hline $\begin{array}{l}\text { gene1663- } \\
\text { D2 }\end{array}$ & Scaffold 157 & 1035 & 1.87 & 0.17 & 1.34 & 0.27 & 3.49 & -3.90 \\
\hline gene9115 & Scaffold2 & 1038 & 111.29 & 4.54 & 2.69 & 21.74 & 10.70 & 0.77 \\
\hline gene1140 & Scaffold 110 & 891 & 5.16 & 4.34 & 2.22 & 5.34 & 4.36 & 0.07 \\
\hline gene4450 & Scaffold 150 & 936 & 101.68 & 38.30 & 6.24 & 29.57 & 22.48 & 0.15 \\
\hline gene2254 & Scaffold201 & 996 & 0.82 & 0.26 & 0.09 & 0.21 & 171.85 & -9.93 \\
\hline $\begin{array}{l}\text { gene10498- } \\
\text { D2 }\end{array}$ & Scaffold62 & 864 & 0.14 & 0.06 & 0.14 & 0.02 & 0.01 & 0.71 \\
\hline $\begin{array}{l}\text { gene9115- } \\
\text { D2 }\end{array}$ & Scaffold 49 & 1038 & 87.98 & 3.11 & 3.31 & 23.40 & 16.45 & 0.25 \\
\hline $\begin{array}{l}\text { gene6325- } \\
\text { D2 }\end{array}$ & Scaffold61 & 1017 & 43.08 & 3.28 & 0.89 & 42.16 & 11.23 & 1.66 \\
\hline
\end{tabular}

\section{Figures}



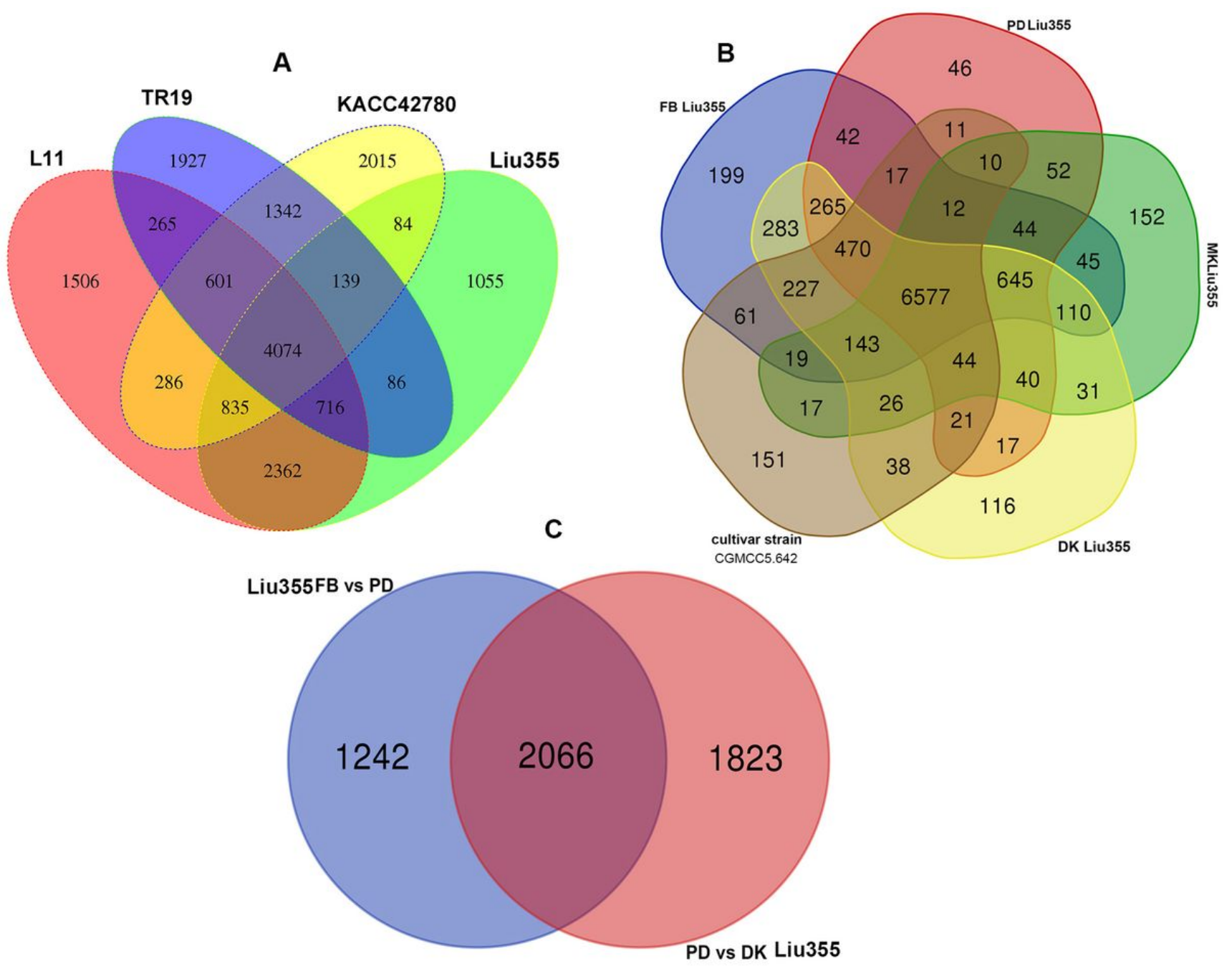

\section{Figure 1}

Fig. 1 Venn diagram showing the numbers of orthologue genes or differentially expressed genes. (A) The numbers of orthologue genes between four strains of F. filiformis. L11(China) in red, TR19 (Japan) in purple, KACC42780 (Korea) in yellow and Liu355 (China) in green. (B). The numbers of differentially expressed genes (DEGs) in various comparative groups of F. filiformis. Fruiting body (FB) in blue, Primordium in red, Monokaryotic mycelium (MK) in green, Dikaryotic mycelium (DK) in yellow and cultivar strain of F. filiformis in brown. (C). Venn diagram showing the numbers of DEGs at adjacent development stage of F. filiformis. Blue color represented the number of DEGs of fruiting body (FB) versus primordium (PD) and red color represented primordium (PD) versus dikaryotic mycelium (DK). Abbreviation: MK: monokaryontic mycelium; DK: dikaryontic mycelium; PD: primordium; FB: fruiting body. 


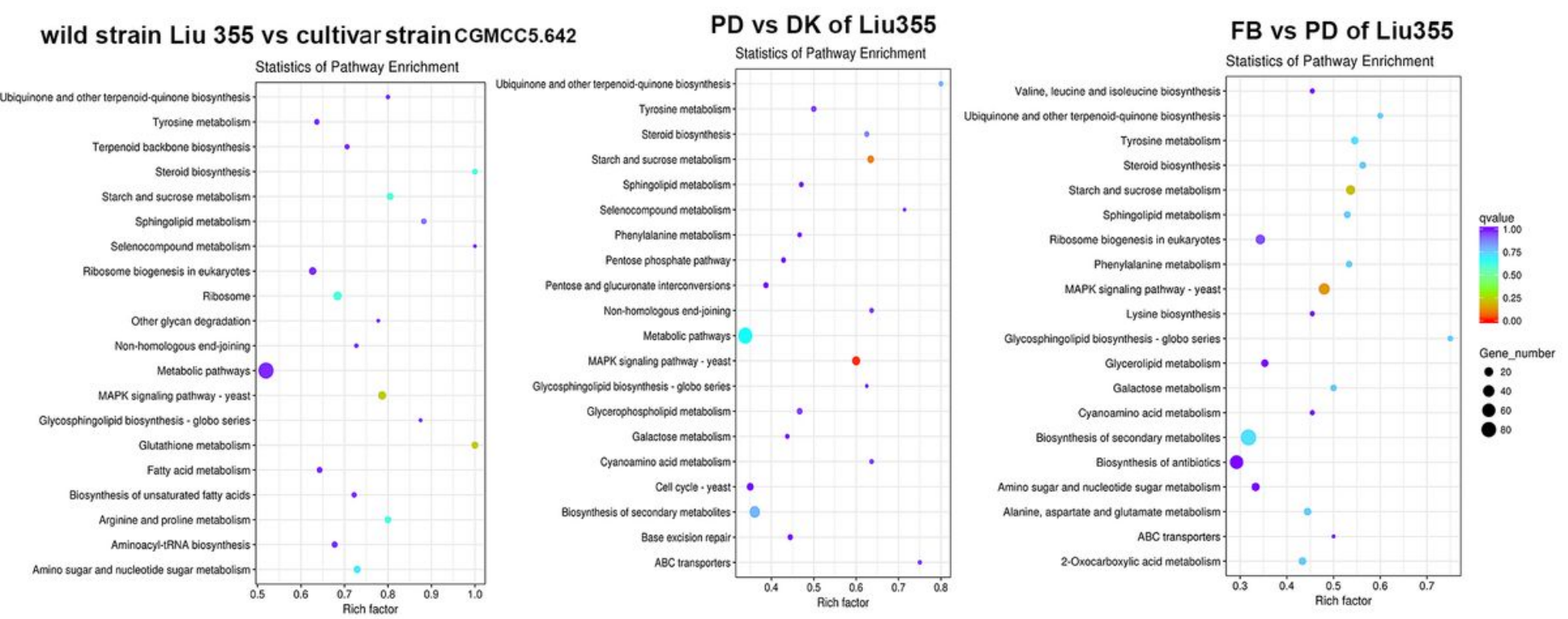

Figure 2

Fig. 2 KEGG pathway enrichment analysis of differentially expressed genes (DEGs) during F. filiformis development. Left columns: pathway enrichment at mycelium stage of wild strain Liu355 compared to cultivar strain CGMCC 5.642; Middle columns: pathway enrichment at primordium stage compared to mycelium stage of wild strain Liu355; Right columns: pathway enrichment at fruiting body stage compared to primordium stage. Abbreviation: MK: monokaryontic mycelium; DK: dikaryontic mycelium; PD: primordium; FB: fruiting body. 


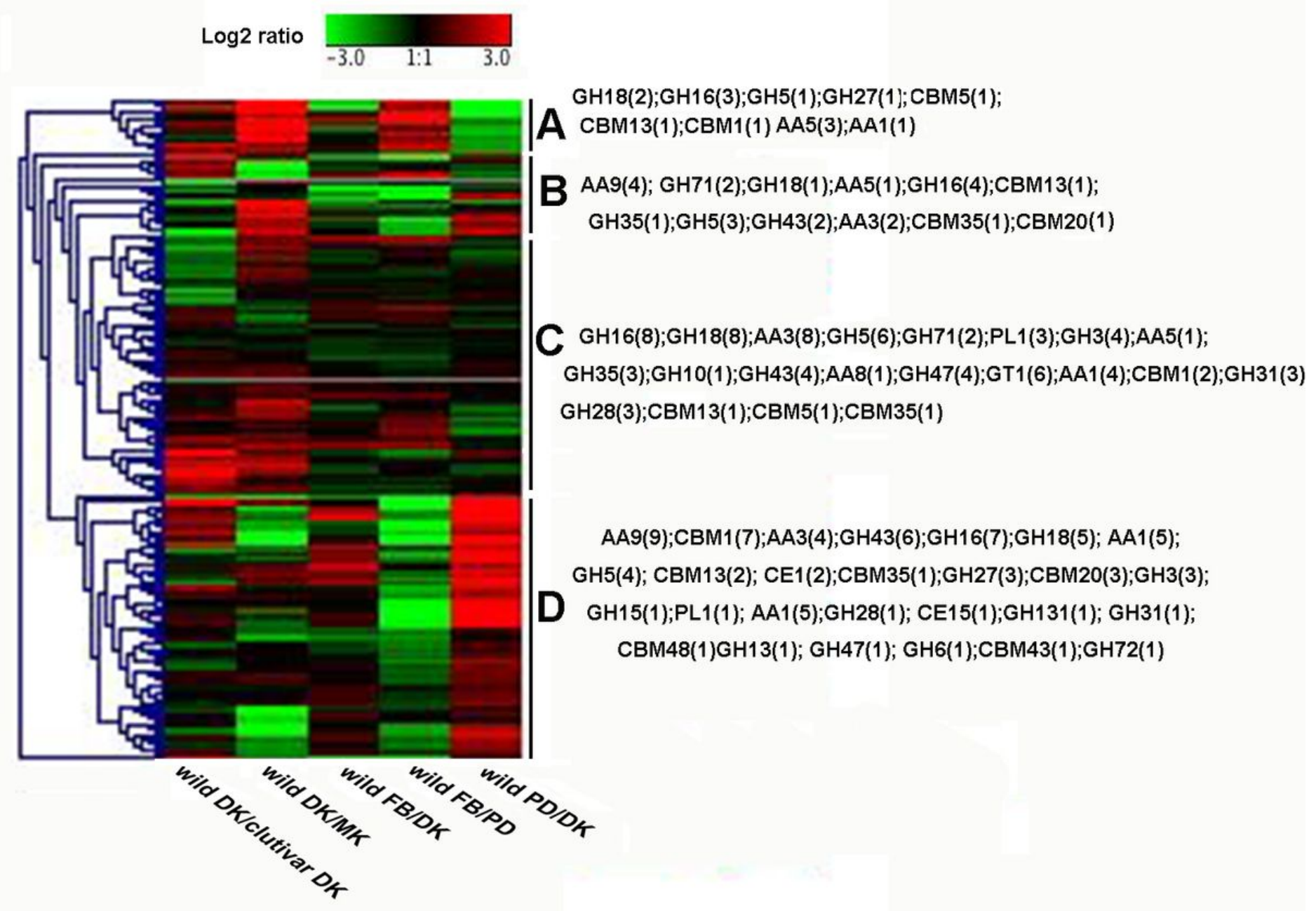

Figure 3

Fig. 3 Hierarchical clustering analysis of 287 CAZy of F. filiformis between wild strain Liu355 and cultivar strain CGMCC 5.642 and among four development stages of wild strain Liu 355. Expression ratios were plotted in a heatmap on a log2 scale. The red and green colors indicate up- and down-regulation, black represents no significant expression change and grey represents missing data The abbreviation: MK, monokaryotic mycelium; DK, Dikaryotic mycelium; PD, primordium; FB, Fruiting body. GH, Glycoside-Hydrolases; AA, Auxiliary Activities containing redox enzymes; GT, glycosyltransferases; CBM Carbohydratebinding module; CE, Carbohydrate Esterases (CE); PL, Polysaccharide Lyases. 


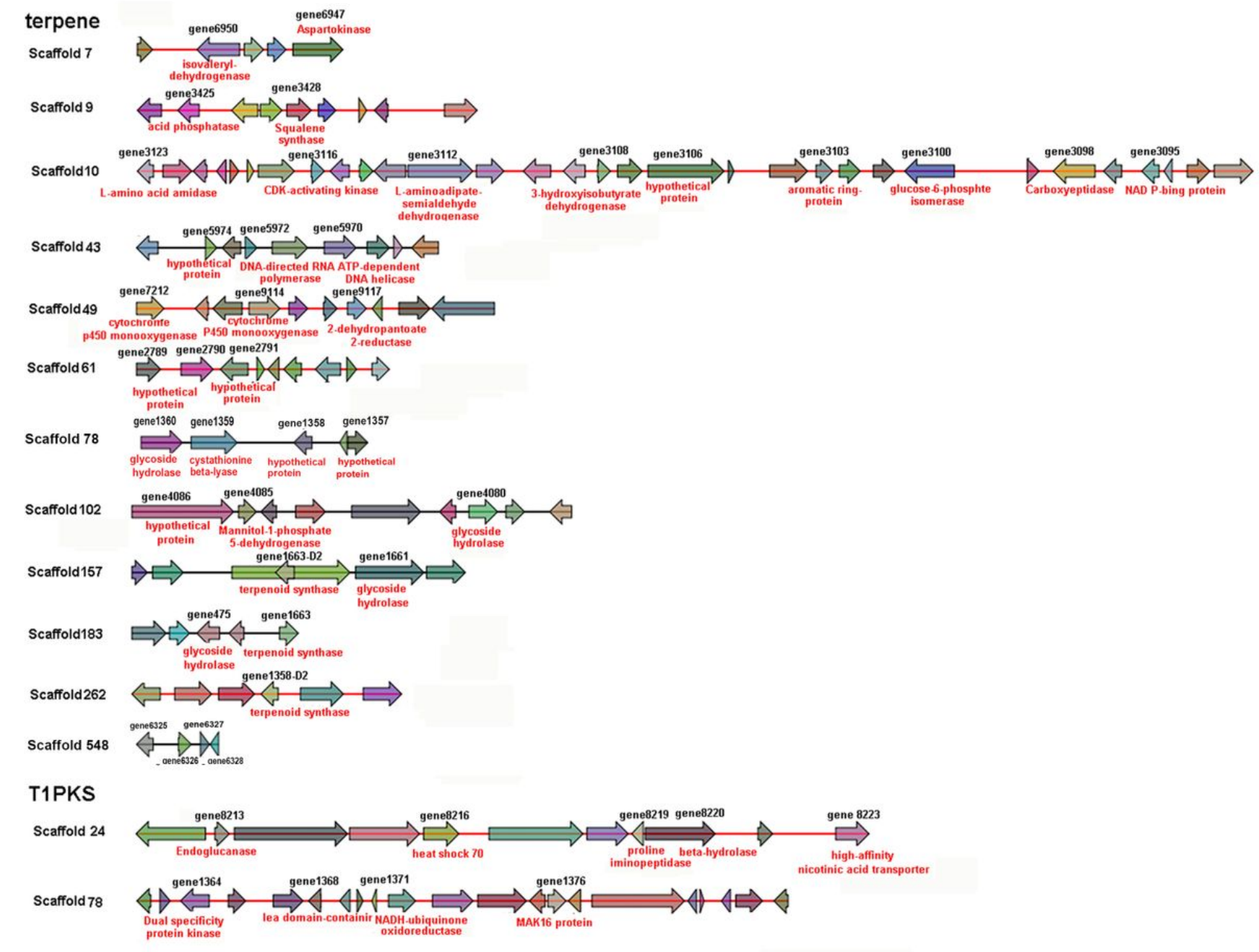

\section{Figure 4}

Fig. 4 Identification of the putative gene clusters for terpene and polyketides (PKS) in F. filiformis genome by antiSMASH software. Genes with SwissProt functional annotation were marked in red color. 


\section{Log2 ratio}

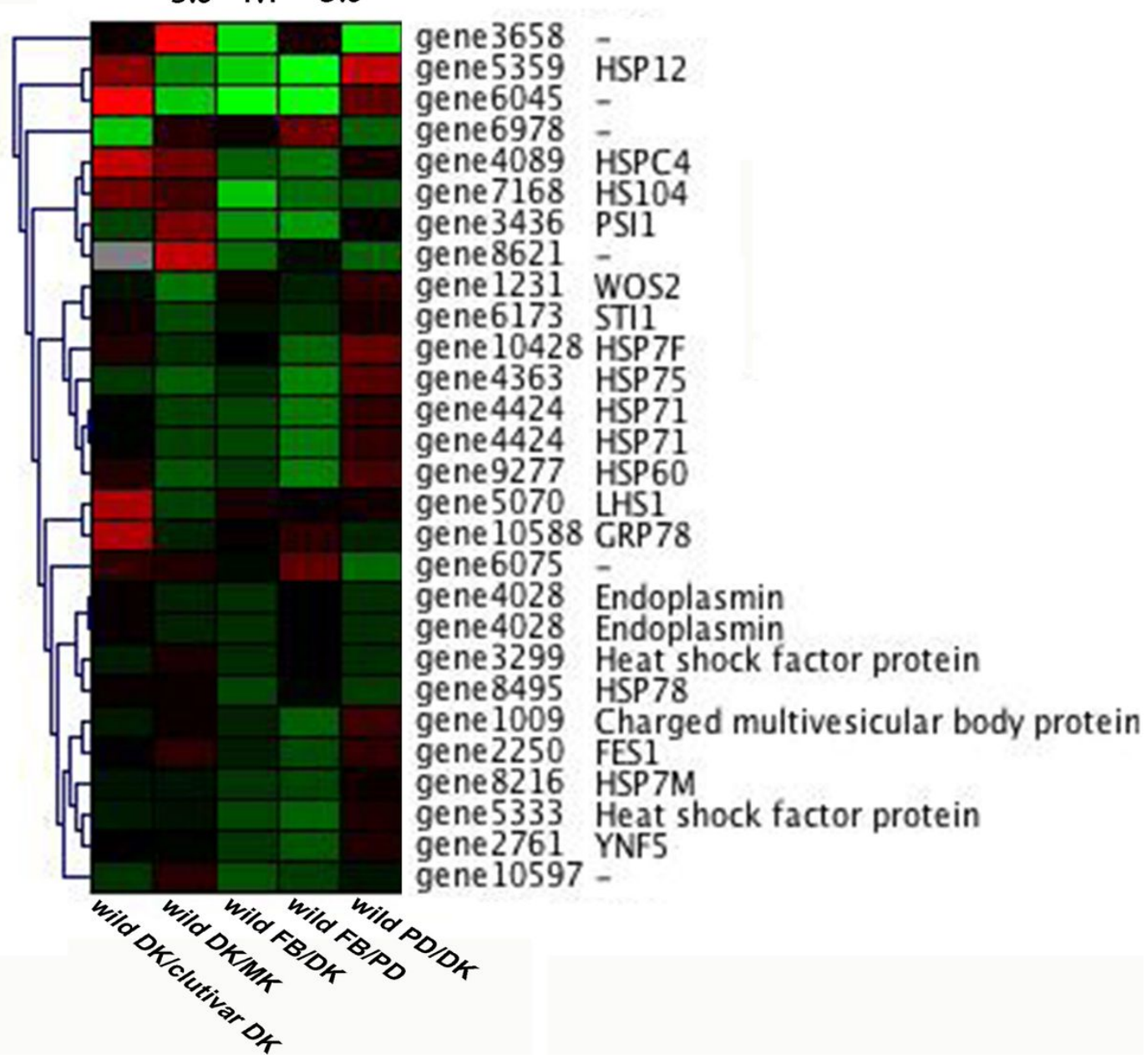

Figure 5

Fig. 5 Hierarchical clustering analysis of 28 putative heat-shock protein encoding genes in F. filiformis genome between wild strain Liu355 and cultivar strain CGMCC5.642 and among four development stages of wild strain Liu 355. Expression ratios were plotted in a heatmap on a log2 scale. The red and green colors indicate up- and down-regulation, black represents no significant expression change and grey represents missing. The abbreviation: MK, monokaryotic mycelium; DK, Dikaryotic mycelium; PD, primordium; FB, Fruiting body.

\section{Supplementary Files}

This is a list of supplementary files associated with this preprint. Click to download.

- supplement1.xlsx

- supplement2.xlsx

- supplement2.xlsx

- supplement4.xlsx

- supplement5.jpg

- supplement6.jpg

- supplement7.docx

- supplement8.jpg

- supplement9.jpg

- supplement10.jpg

- supplement11.jpg

- supplement12.docx 